\title{
Sistemas de automatización en unidades LACT para la medición dinámica de hidrocarburos en la industria petrolera
}

DOI: https://doi.org/10.33262/ap.v3i3.1.73

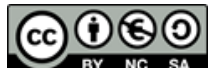

Automation systems in LACT units for dynamic measurement of hydrocarbons in the oil industry

Andrés David Padilla Romero. ${ }^{1}$, Sayda Cecilia Chamba Melo. ${ }^{2}$, Gabriela Cristina Aguilera Flores. ${ }^{3} \&$ Hilda Fabiola Anguaya Isama. ${ }^{4}$

\begin{abstract}
.
The paper aims to present automation systems in LACT units for the dynamic measurement of hydrocarbons in the oil industry, due to the importance of their instruments in the processes of custody transfer (CT) of hydrocarbons. For this, concepts about dynamic measurement in the hydrocarbon industry were developed. Subsequently, three automation systems are presented in LACT units for the dynamic measurement of gas in a two-line station, naphtha and diesel in the Pozos Colorados station, and oil in the complex of production and facilities Indillana of the Petroamazonas EP, each system contains a P\&ID and the instruments for its automation and control, Emerson and Siemens brand flow meters also shown. Systematized tables were prepared from the P\&ID, which show the industrial instruments and their connectivity, through SCADA, with the control instruments, as a guide to design automation systems for LACT units.
\end{abstract}

\footnotetext{
${ }^{1}$ Ingeniero electrónico en Automatización y Control, Instituto Superior Pedagógico Martha Bucaram de Roldós - Bilingüe Intercultural, apadilla@institutos.gob.ec, https://orcid.org/0000-0002-2547-6545

${ }^{2}$ Ingeniería en Informática y sistemas computacionales, Instituto Superior Pedagógico Martha Bucaram de Roldós - Bilingüe Intercultural, schamba@institutos.gob.ec, https://orcid.org/0000-0003-2104-2114

${ }^{3}$ Licenciatura en Ciencias de la Educación, mención plurilingüe, Instituto Superior Pedagógico Martha Bucaram de Roldós - Bilingüe Intercultural, gaguilera@institutos.gob.ec, https://orcid.org/0000-00024831-2519

${ }^{4}$ Ingeniería en Ambiente y Desarrollo, Instituto Superior Pedagógico Martha Bucaram de Roldós Bilingüe Intercultural, hanguaya@institutos.gob.ec, https://orcid.org/0000-0002-6142-4961
} 
The correct dynamic measurement of gas, naphtha, diesel and oil, for the CT process, by the automation systems presented, depend on the standard linearity and repeatability of the flow meter, the development of computer networks and networks communication through field buses, these last two concepts are within what is called automation 4.0.

Keywords: LACT automation systems, LACT units hydrocarbons, Dynamic measurement hydrocarbons, P\&ID of LACT units hydrocarbons, LACT unit instruments hydrocarbons.

\section{Resumen.}

El artículo tiene por objetivo presentar sistemas de automatización en unidades LACT para la medición dinámica de hidrocarburos en la industria petrolera, debido a la importancia que tienen sus instrumentos en los procesos de transferencia de custodia (TC) de hidrocarburos. Para esto, se elaboró conceptos relacionados a la medición dinámica en la industria de hidrocarburos. Posteriormente se presentan tres sistemas de automatización en unidades LACT para la medición dinámica de gas en una estación de dos líneas, nafta y diésel en la estación Pozos Colorados, y petróleo en el complejo de producción y facilidades Indillana de Petroamazonas EP, cada sistema contiene un P\&ID y los instrumentos para su automatización y control, también se muestran medidores de caudal de la marca Emerson y Siemens. A partir del P\&ID se elaboró tablas sistematizadas que muestran los instrumentos industriales y su conectividad, mediante SCADA, con los instrumentos de control, como una guía para diseñar sistemas de automatización de unidades LACT. La correcta medición dinámica de gas, nafta, diésel y petróleo, para el proceso de TC, por parte de los sistemas de automatización presentados, va a depender de la repetibilidad y linealidad estándar del medidor de flujo, el desarrollo de las redes informáticas y de las redes de comunicación mediante buses de campo, estos dos últimos conceptos están dentro de lo que se denomina automatización 4.0 .

Palabras claves: Sistemas de automatización LACT, unidades LACT de hidrocarburos, medición dinámica de hidrocarburos, P\&ID en unidades LACT de hidrocarburos, instrumentos en unidades LACT de hidrocarburos.

\section{Introducción}

Los hidrocarburos, tanto en el Ecuador como en el mundo, son una fuente de ingresos económicos para el presupuesto general de un estado y ganancias económicas en el caso de empresas con fines de lucro. Estos hidrocarburos como el gas, gasolina, diésel, nafta, petróleo, entre otros, para su comercialización, atraviesan un proceso de transferencia de custodia (TC), en donde se debe asegurar que la calidad y cantidad del hidrocarburo, medido y transferido, sea la estipulada en el contrato. Esto da origen a que la medición dinámica de hidrocarburos debe ser confiable y precisa, ya que un error, sea de decimas o centésimas, generaría graves consecuencias económicas, tanto al vendedor como al comprador. 
La TC en la industria de hidrocarburos ha originado que la medición dinámica del fluido en una tubería u oleoducto sea estandarizada, lo que ha permitido el desarrollo de las denominadas unidades LACT (Lease Automatic Custody Transfer). Estas unidades se utilizan para la medición de flujo de hidrocarburos, siendo diseñadas y fabricadas de acuerdo a los estándares API, siendo su principal componente el medidor de flujo.

El constante desarrollo de la tecnología mejora la automatización y control de las unidades LACT, a tal punto que los nuevos conceptos de industria 4.0, automatización 4.0, redes de comunicación industrial, etc., simplifican su funcionamiento y potencian su rendimiento.

La investigación propuesta busca contribuir con un análisis de la instrumentación y redes industriales utilizadas en los sistemas de automatización para transferencia de custodia de hidrocarburos, revisando e ilustrando los conceptos involucrados.

En el desarrollo de este tema de investigación se siguió una metodología inductivasintética, fundamentándose en conceptos desarrollados por fuentes investigativas para elaborar una síntesis y presentar un nuevo conocimiento. El artículo se fundamentó en la revisión y análisis de investigaciones como las desarrolladas por Escobar et al. (2018) Instrumentación para sistemas automatizados de medición dinámica de hidrocarburos. Jiménez et al. (2013) Ingeniería de detalle para ampliación y automatización del proceso de bombeo en pozos petroleros. Falcón (2017) Optimización del proceso de deshidratación del crudo en la planta de tratamiento del complejo de producción y facilidades Indillana (CPF) de Petroamazonas EP. Siemens (2021) Productos para la instrumentación de procesos, entre otras.

\section{Medición dinámica en la industria de hidrocarburos}

El desarrollo a nivel industrial de los hidrocarburos exigió como parte inicial su correcta medición dinámica, por esta razón, para garantizar que la cantidad se ajuste a la realidad, se crearon conceptos como la transferencia de custodia (TC), medición dinámica, entre otros, para dar paso al diseño y fabricación de sistemas de medición dinámica para procesos de TC, conocidas en la industria petrolera como unidades LACT, siendo el medidor de flujo uno de los elementos de alta importancia del mismo.

\section{Transferencia de custodia (TC)}

La TC es un proceso de compraventa de un hidrocarburo, donde el actual propietario cede toda la posesión, el control y la responsabilidad de su manejo a un nuevo propietario. Este proceso de intercambio utiliza sistemas de instrumentación industrial para asegurar que la calidad y cantidad del hidrocarburo medido y transferido sea la estipulada en el contrato. El concepto de TC asegura que las mediciones de caudal de un hidrocarburo sean de alta precisión, ya que, tiene propósitos fiscales (Escobar et al., 2018) (Lipták y Venczel, 2016). 


\section{Medición dinámica}

La medición dinámica de hidrocarburos es un proceso que requiere de la instrumentación, automatización y control industrial, para calcular, registrar y visualizar con exactitud, y en tiempo real, el caudal que fluye por la tubería. Para la medición se realiza la evaluación de variables físicas de temperatura, presión y velocidad (Escobar et al., 2018) (Sánchez, 2010).

\section{Sistemas de medición dinámica para transferencia de custodia}

Los sistemas de medición dinámica tienen aplicaciones en los procesos de transferencia de custodia, estos sistemas contienen elementos de instrumentación industrial que permiten la compraventa del hidrocarburo con exactitud y a través de una sola medida. En la industria petrolera los sistemas de medición dinámica para TC se realizan mediante las denominadas unidades LACT (Lease Automatic Custody Transfer), estas unidades son diseñadas y construidas siguiendo los lineamientos del Instituto Americano del Petróleo (API) (Escobar et al., 2018) (Sánchez, 2010).

\section{Unidades LACT (Lease Automatic Custody Transfer)}

Los conceptos básicos como LACT y unidad LACT han sido definidos, debido a que tienen relación con la medición dinámica de hidrocarburos. Para el diseño y fabricación de las unidades LACT se utilizan normas y estándares internacionales.

\section{LACT}

Las siglas LACT significan Lease Automatic Custody Transfer y son utilizadas en la industria de los hidrocarburos para procesos de TC, siendo su significado en español Fiscalización Automática para la Transferencia de Custodia.

\section{Unidad LACT}

La unidad LACT es un sistema constituido por elementos de instrumentación industrial; como mecánicos, eléctricos, electrónicos, informáticos y redes de comunicación industrial; que miden y registran automáticamente la TC de hidrocarburos, producidos en campo o almacenados en tanques de reserva, conducidos por tubería u oleoducto a su lugar de destino, su estructura permite que el hidrocarburo cumpla con parámetros de calidad antes de ingresar al caudalímetro y que el flujo sea medido con precisión (Escobar et al., 2018) (Lipták y Venczel, 2016) (ABUIN GARCÍA OIL, 2016).

\section{Elementos de una unidad LACT}

La Figura 1 muestra los elementos de una unidad LACT para medición de caudal de hidrocarburos, en este caso la unidad LACT está conectada por medio de una bomba de carga al tanque de almacenamiento. En la zona de bombeo está ubicada la bomba de carga que realiza la succión, a través de la tubería, del hidrocarburo que está en el tanque, y lo envía a la zona de filtrado, en donde se verifica la calidad del mismo, seguidamente, el 
hidrocarburo pasa a la zona de medición con el objetivo de que sea medido su caudal, el instrumento utilizado en la medición dinámica es un medidor de flujo con control industrial. Una vez registrado el caudal del hidrocarburo su flujo se interconecta a la estación de bombeo principal para su despacho por tubería u oleoducto (FMC Technologies, 2012).

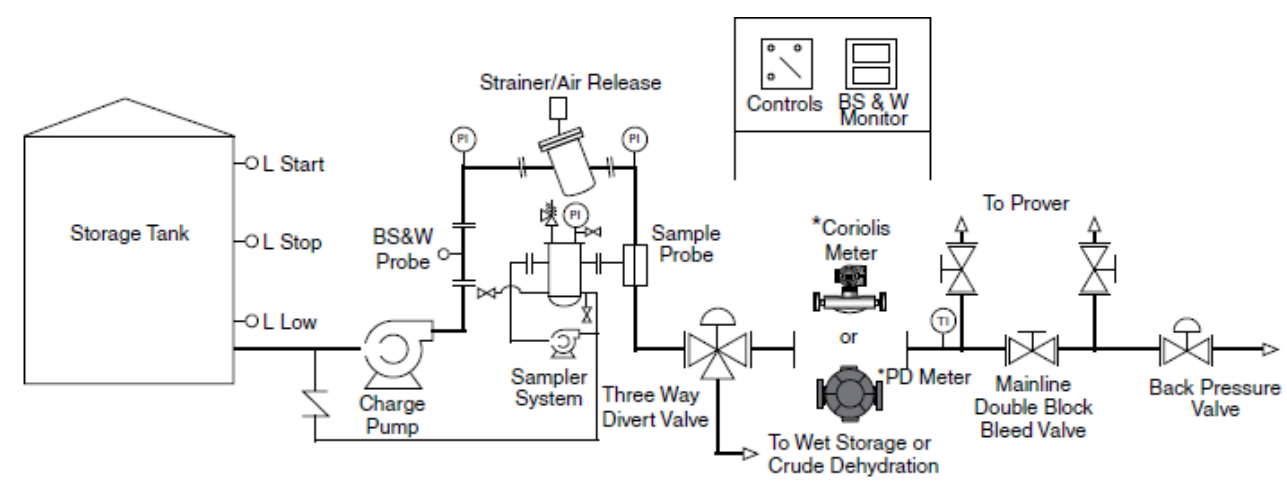

Figura 1. Unidad LACT para medición del caudal de hidrocarburos (FMC Technologies, 2012)

Dependiendo de los requerimientos para el proceso de TC las unidades LACT varían en su tamaño, diseño y configuración. Básicamente las unidades LACT están conformadas por los elementos de la Figura 1, siendo sus principales elementos (FMC Technologies, 2012):

Bomba de carga (Charge Pump) Sistema de maestro (Sampler System)

Filtro y liberador de aire (Strainer/Air Release) Sonda de muestra y monitor de variables físicas (BS\&W Probe and Monitor)
Válvula de desvió (Divert Valve)

Medidor de caudal (Meter)

Válvula de prueba (Prover Valve)

Válvula de control de contrapresión (Back Pressure Control Valve)

\section{Normas y estándares utilizados para el diseño y fabricación de unidades LACT}

En el Ecuador las normas utilizadas para el diseño y fabricación de unidades LACT en la industria de hidrocarburos son las citadas a continuación:

Norma ASME/ANSI B31.3 “Chemical Plants and Petroleum Refinery Piping”.

Norma API Specification for Lease Automatic Custody Transfer (LACT) Equipment.

Norma API Manual of Petroleum Measurement Standards: Metering Assemblies Section 1 Lease Automatic Custody Transfer (LACT) Systems.

\section{Medidores de flujo}

Un medidor de flujo es un instrumento utilizado para la medición de caudal, gasto volumétrico o gasto másico de algún tipo de fluido. Este instrumento es montado en línea con la tubería que transporta el hidrocarburo. Los medidores de flujo suelen denominarse caudalímetros, medidores de caudal o flujómetros. 
El medidor de flujo es el elemento de mayor importancia en la TC de hidrocarburos, ya que sin una correcta medición sería imposible o existiría errores al momento de facturar la compraventa. Para la medición de flujo en procesos de TC el medidor interactúa con sensores, transmisores, transductores y base de datos, que evalúan las variables físicas como viscosidad, densidad, gravedad específica, compresibilidad, velocidad, temperatura y presión del fluido, estas variables son analizadas por un computador de control para calcular la cantidad de fluido que pasa por la tubería en un intervalo de tiempo (caudal).

Para la transferencia de custodia de hidrocarburos existen varios tipos de medidores de flujos que son utilizados según los requerimientos de la industria. En la Figura 2 se presenta una clasificación de los medidores de flujo.

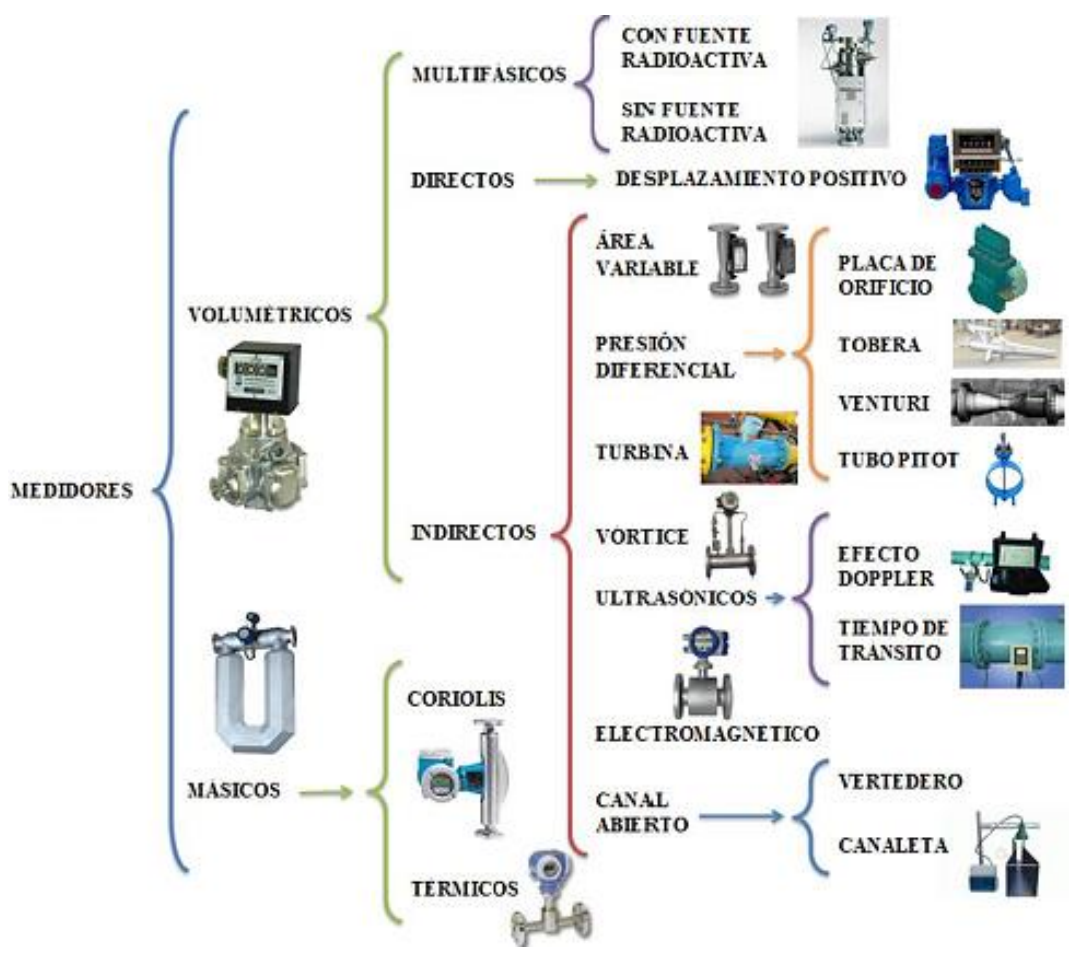

Figura 2. Clasificación de los medidores de flujo (Arequipa y Loyo, 2013)

\section{Sistemas de automatización en unidades lact}

Se presentan tres sistemas de automatización en unidades LACT para la medición dinámica de gas en una estación de dos líneas, nafta y diésel en la estación Pozos Colorados, y petróleo en complejo de producción y facilidades Indillana de Petroamazonas EP. En cada sistema se muestra un diagrama de tuberías e instrumentación y los instrumentos para la automatización y control. Al ser el medidor de caudal el elemento de mayor importancia en una unidad LACT se ha expuesto medidores de las marcas Emerson y Siemens. Como parte de un análisis se presentan resultados obtenidos de la investigación. 
Sistema de automatización en una unidad LACT para la medición dinámica de gas en una estación de dos líneas

A continuación, se presenta el diagrama de P\&ID y los instrumentos para la automatización y control de la unidad LACT para la medición dinámica de gas en una estación de dos líneas.

\section{Diagrama de tuberías e instrumentación (P\&ID) de la unidad LACT para la medición dinámica de gas}

En la Figura 3 se muestra el P\&ID de la unidad LACT para la medición dinámica del caudal de gas. El P\&ID sirve como guía para realizar los primeros pasos en la automatización y control de los elementos que conforman la unidad LACT, además de, permitir la correcta medición de caudal de gas en la estación de dos líneas. El P\&ID cuenta con dos líneas para el flujo de gas, siendo la conformación de instrumentos de la primera línea igual a la segunda.

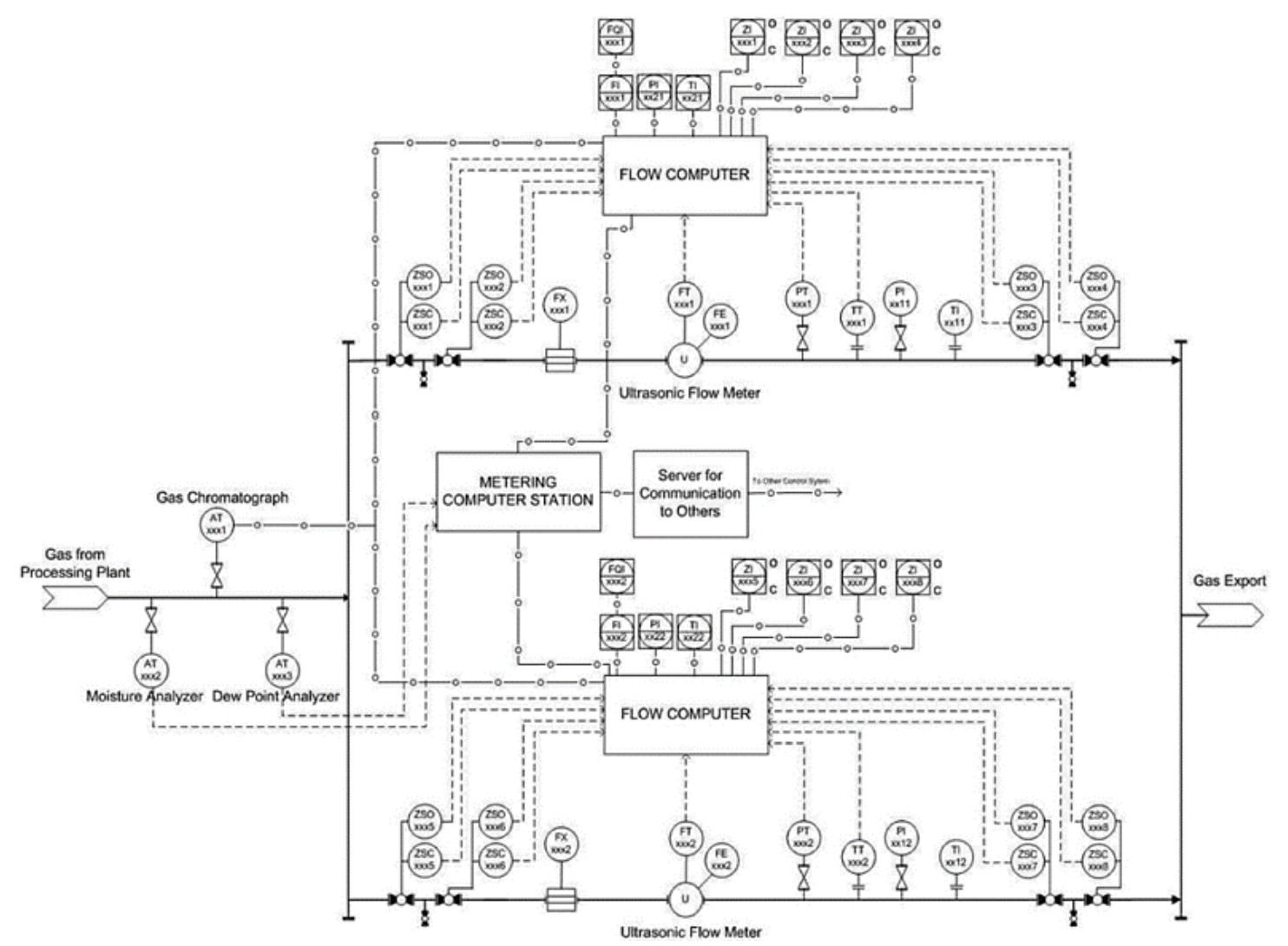

Figura 3. P\&ID de la unidad LACT para la medición dinámica de caudal de gas (Escobar et al., 2018)

Una vez que el gas ha sido tratado en la planta de procesamiento este pasa al comienzo de la tubería de la estación para iniciar la fase de filtrado. En la fase de filtrado están colocados el transmisor de análisis de cromatografía, transmisor de análisis de humedad y transmisor de análisis de punto de rocío, que aseguran que la calidad del gas este dentro 
de los parámetros establecidos. Posteriormente, el gas ingresa a la fase de estabilización, en la cual, el gas pasa por las válvulas de bola, que son controladas por los sensores de posición ZSO XXX1, ZSC XXX1, ZSO XXX2 y ZSC XXX2, y entra al convertidor FX para estabilizar su flujo, una vez estabilizado el gas, el mismo ingresa a la fase de medición. En la fase de medición se realiza el proceso de TC del hidrocarburo mediante un medidor de flujo ultrasónico con indicador FE incorporado y un transmisor de flujo FT para calcular el caudal del fluido, más adelante se encuentra un medidor de presión, transmisor de temperatura, manómetro de presión, indicador de temperatura; y válvulas de bola controladas por los sensores de posición ZSO XXX3, ZSC XXX3, ZSO XXX4 y ZSC XXX4, para el despacho de gas por la tubería.

Todos los sensores y transmisores están comunicados con el computador de flujo y este a su vez con la estación informática de medición. Esta comunicación es de gran importancia, ya que, es aquí donde se interpretan las variables físicas de viscosidad, densidad, gravedad específica, compresibilidad, velocidad, temperatura y presión del fluido, para calcular y registrar la cantidad de fluido que pasa por la tubería.

\section{Instrumentos para la automatización y control de la unidad LACT para medición dinámica de gas}

En la Tabla 1 se muestran los instrumentos que integran el sistema de automatización y control de la unidad LACT para la medición dinámica de gas en una estación de dos líneas.

En la parte superior izquierda de la Tabla 1 están los instrumentos de campo y panel, los cuales, interactúan con los instrumentos de campo de variable del proceso. En la parte superior derecha se establece la conectividad entre los instrumentos de campo con los instrumentos de control mediante el sistema de Supervisión, Control y Adquisición de Datos (SCADA). La Tabla 1 indica si el instrumento está montado en campo, mientras que su lazo de control lo identifica en el sistema de automatización. Como la primera línea de la estación de gas es idéntica a la segunda, los instrumentos industriales de la segunda línea son los mismos de la Tabla 1.

Tabla 1. Instrumentos del sistema de automatización y control de la unidad LACT para medición dinámica de gas en una estación de dos líneas

\begin{tabular}{|c|c|c|c|c|c|c|}
\hline \multirow{2}{*}{$\begin{array}{c}\text { Instrumentos de campo } \\
\text { y de panel (I) }\end{array}$} & \multirow[b]{2}{*}{ Fase } & \multirow{2}{*}{$\begin{array}{l}\text { Instrumentos de campo de } \\
\text { variable del proceso } \\
\text { conectados a los } \\
\text { instrumentos de campo o de } \\
\text { panel }\end{array}$} & \multicolumn{2}{|c|}{$\begin{array}{c}\text { Supervisión, Control y Adquisición } \\
\text { de Datos (SCADAA) }\end{array}$} & \multirow{2}{*}{$\begin{array}{l}\text { Ubicado } \\
\text { en } \\
\text { campo }\end{array}$} & \multirow{2}{*}{$\begin{array}{l}\text { Lazo de } \\
\text { control }\end{array}$} \\
\hline & & & $\begin{array}{l}\text { Computador } \\
\text { de flujo }\end{array}$ & $\begin{array}{c}\text { Estación } \\
\text { informática de } \\
\text { medición }\end{array}$ & & \\
\hline Válvula de compuerta 1 & $\mathrm{FF}$ & $\begin{array}{l}\text { AT Transmisor de análisis de } \\
\text { cromatografía de gas }\end{array}$ & & AT & I-AT & XXX1 \\
\hline Válvula de compuerta 2 & $\mathrm{FF}$ & $\begin{array}{l}\text { AT Transmisor de análisis de } \\
\text { humedad }\end{array}$ & AT & & & $\mathrm{XXX} 2$ \\
\hline Válvula de compuerta 3 & $\mathrm{FF}$ & $\begin{array}{l}\text { AT Transmisor de análisis de } \\
\text { punto de rocío }\end{array}$ & AT & & I-AT & XXX3 \\
\hline Válvula de bola 1 & $\mathrm{FE}$ & $\begin{array}{l}\text { ZSC Sensor de posición } \\
\text { cerrado } \\
\text { ZSO Sensor de posición } \\
\text { abierto }\end{array}$ & ZSC-ZSO & & $\begin{array}{l}\text { I-ZSC- } \\
\text { ZSO }\end{array}$ & $\begin{array}{l}\mathrm{XXX1} \\
\mathrm{XXX1}\end{array}$ \\
\hline Válvula de bola 2 & $\mathrm{FE}$ & $\begin{array}{l}\text { ZSC Sensor de posición } \\
\text { cerrado } \\
\text { ZSO Sensor de posición } \\
\text { abierto }\end{array}$ & ZSC-ZSO & & $\begin{array}{l}\text { I-ZSC- } \\
\text { ZSO }\end{array}$ & $\begin{array}{l}\text { XXX2 } \\
\text { XXX2 }\end{array}$ \\
\hline
\end{tabular}




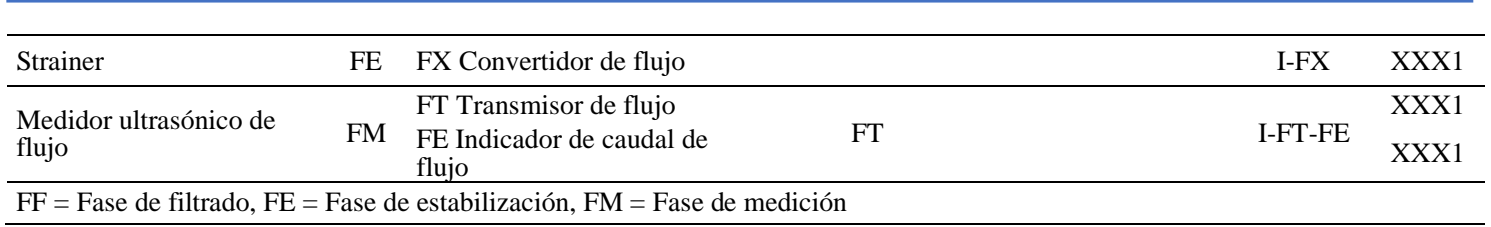

Fuente: Elaboración propia basada en la Figura 3

Tabla 1. Continuación

\begin{tabular}{|c|c|c|c|c|c|c|}
\hline \multirow{2}{*}{$\begin{array}{c}\text { Instrumentos de campo y } \\
\text { de panel (I) }\end{array}$} & \multirow[b]{2}{*}{ Fase } & \multirow{2}{*}{$\begin{array}{c}\text { Instrumentos de variable } \\
\text { del proceso conectados a los } \\
\text { instrumentos de campo o de } \\
\text { panel }\end{array}$} & \multicolumn{2}{|c|}{$\begin{array}{c}\text { Supervisión, Control y Adquisición } \\
\text { de Datos (SCADA) }\end{array}$} & \multirow{2}{*}{$\begin{array}{l}\text { Ubicado } \\
\text { en } \\
\text { campo }\end{array}$} & \multirow{2}{*}{$\begin{array}{l}\text { Lazo de } \\
\text { control }\end{array}$} \\
\hline & & & $\begin{array}{l}\text { Computador } \\
\text { de flujo }\end{array}$ & $\begin{array}{c}\text { Estación } \\
\text { informática de } \\
\text { medición }\end{array}$ & & \\
\hline $\begin{array}{l}\text { Válvula de compuerta para } \\
\text { acoplar transmisor de } \\
\text { presión }\end{array}$ & FM & PT Transmisor de presión & PT & & I-PT & XXX1 \\
\hline $\begin{array}{l}\text { Transmisor de temperatura } \\
\text { TT }\end{array}$ & FM & & I & & I & XXX1 \\
\hline $\begin{array}{l}\text { Válvula de compuerta para } \\
\text { toma de presión }\end{array}$ & FM & PI Manómetro de presión & & & I-PI & XX11 \\
\hline Indicador de temperatura TI & FM & & & & I & XX11 \\
\hline Válvula de bola 3 & FM & $\begin{array}{l}\text { ZSC Sensor de posición } \\
\text { cerrado } \\
\text { ZSO Sensor de posición } \\
\text { abierto }\end{array}$ & ZSC-ZSO & & $\begin{array}{l}\text { I-ZSC- } \\
\text { ZSO }\end{array}$ & $\begin{array}{l}\text { XXX3 } \\
\text { XXX3 }\end{array}$ \\
\hline Válvula de bola 4 & FM & $\begin{array}{l}\text { ZSC Sensor de posición } \\
\text { cerrado } \\
\text { ZSO Sensor de posición } \\
\text { abierto }\end{array}$ & ZSC-ZSO & & $\begin{array}{l}\text { I-ZSC- } \\
\text { ZSO }\end{array}$ & $\begin{array}{l}\text { XXX4 } \\
\text { XXX4 }\end{array}$ \\
\hline Display indicador de caudal & FM & FI Indicador de caudal & I-FI & & & XXX1 \\
\hline $\begin{array}{l}\text { Display indicador total de } \\
\text { caudal }\end{array}$ & FM & $\begin{array}{l}\text { FQI Indicador total de caudal } \\
\text { conectado con FI }\end{array}$ & & & & XXX1 \\
\hline Display de presión & FM & PI Indicador de presión & I-PI & & & XX21 \\
\hline Display de temperatura & FM & TI Indicador de temperatura & $\mathrm{I}-\mathrm{TI}$ & & & $\mathrm{XX} 21$ \\
\hline Display de indicador & FF & $\begin{array}{l}\text { ZI Indicador de posición de la } \\
\text { válvula de bola } 1\end{array}$ & I-TI & & & XXX1 \\
\hline Display de indicador & $\mathrm{FF}$ & $\begin{array}{l}\text { ZI Indicador de posición de la } \\
\text { válvula de bola } 2\end{array}$ & I-TI & & & $\mathrm{XXX} 2$ \\
\hline Display de indicador & FM & $\begin{array}{l}\text { ZI Indicador de posición de la } \\
\text { válvula de bola } 3\end{array}$ & I-TI & & & XXX3 \\
\hline Display de indicador & FM & $\begin{array}{l}\text { ZI Indicador de posición de la } \\
\text { válvula de bola } 4\end{array}$ & I-TI & & & $\mathrm{XXX} 4$ \\
\hline Computadora de flujo & FM & & & I & & \\
\hline $\begin{array}{l}\text { Servidor de comunicación } \\
\text { externa }\end{array}$ & FM & & & I & & \\
\hline
\end{tabular}

Fuente: Elaboración propia basada en la Figura 3

\section{Sistema de automatización en una unidad LACT para la medición dinámica de nafta y diésel en la estación Pozos Colorados}

El proyecto de aumento de la capacidad de bombeo de nafta y diésel desde la estación Pozos Colorados, ubicada en la ciudad de Santa Marta Colombia, hacia la estación Galan de Barrancabermeja, tuvo como una de sus mejoras, mediante la ingeniería de detalle desarrollada por Jiménez et al. (2013), la adición de una unidad LACT en la estación, con lo cual, los hidrocarburos fueron bombeados y transportados con un mayor flujo, a través del poliducto. A partir del proyecto se detalló para esta investigación el diagrama de tuberías e instrumentación y los instrumentos para la automatización y control de la unidad LACT. 


\section{Diagrama de tuberías e instrumentación (P\&ID) de la unidad LACT para la medición dinámica de nafta y diésel}

La unidad LACT es un diseño original de la estación Pozos Colorados, pero como se mencionó anteriormente, en la ingeniería de detalle se determinó que solo era necesario añadir una unidad y ciertas mejoras que no modifican el diseño original. En la Figura 4 se muestra el P\&ID de la unidad LACT para la medición dinámica de caudal de nafta y diésel, el diseño de la unidad es un estándar que la integran la fase de bombeo, la fase de filtrado y la fase de medición. El P\&ID sirve como guía para realizar los primeros pasos en la automatización y control de los elementos que conforman la unidad LACT, además de, permitir la correcta medición de caudal en los hidrocarburos nafta y diésel de la estación Pozos Colorados (Jiménez et al., 2013).

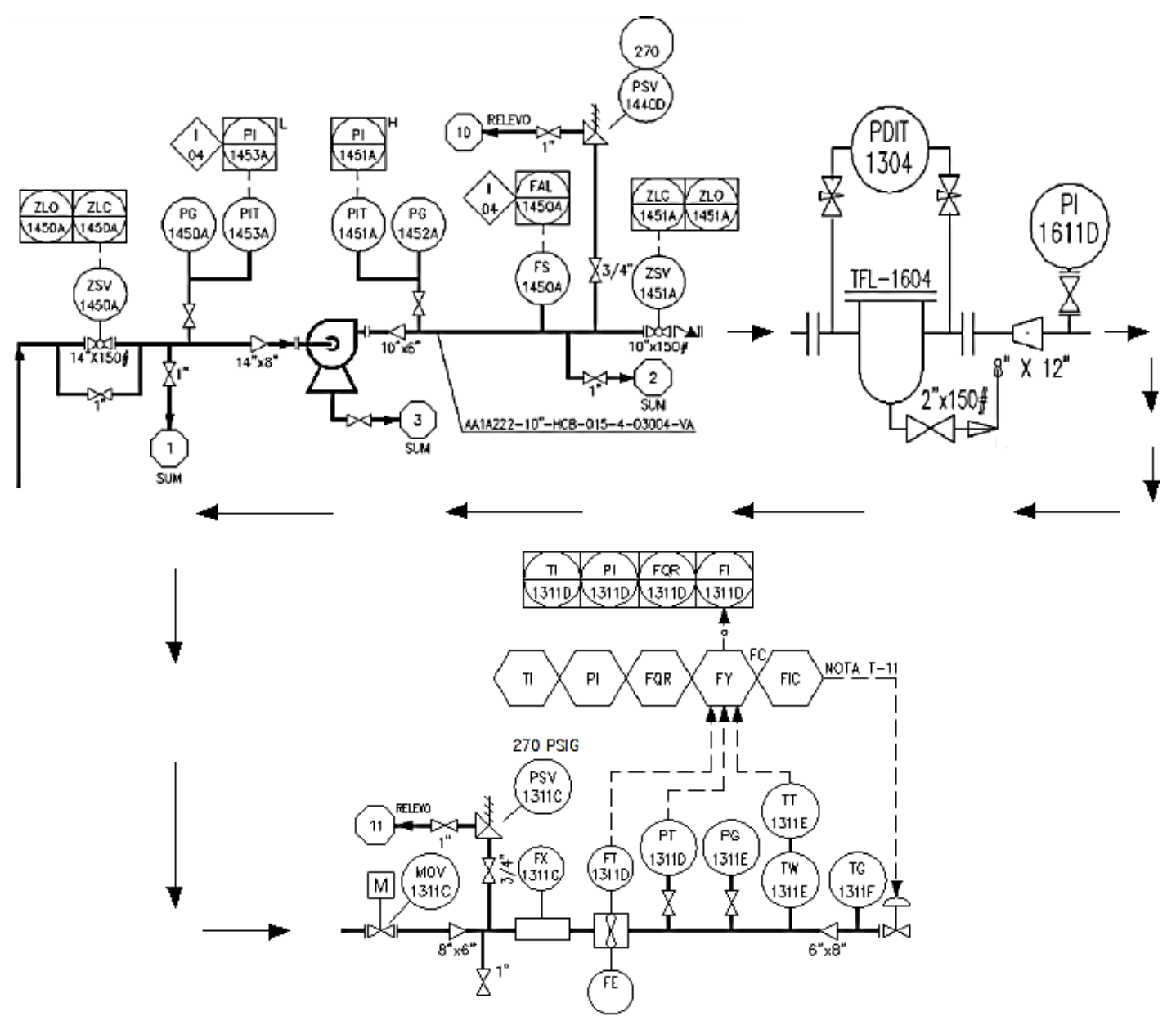

Figura 4. P\&ID de la unidad LACT para la medición dinámica de caudal de nafta y diésel (Jiménez et al., 2013)

La primera fase es el sistema de bombeo que consta de una bomba booster que succiona el hidrocarburo por la válvula de bola de entrada la misma posee un sensor de posición ZSV 1450A para detectar su estado. También se tiene un lazo de control de baja presión (L) para succión a la entrada de la bomba, de igual forma, se instaló un lazo de control de presión alta $(\mathrm{H})$ en la descarga de la bomba booster. Existe un sensor de flujo todo o nada FS 1450A para el control del caudal. Por seguridad se asoció una válvula de alivio de 
presión con regulador PSV 1440D. A la salida del sistema se incorporó una válvula de bola que posee un sensor de posición ZSV 1451A para indicar su estado (Jiménez et al., 2013).

La segunda fase es el sistema de filtrado que consta de un filtro tipo canasta TFL-1604 que garantiza la calidad del hidrocarburo. El sistema tiene integrado un transmisor indicador de presión diferencial PDIT 1304 que sirve para detectar si el filtro está deteriorado, también se tiene un indicador de presión PI 1611D a la salida del sistema (Jiménez et al., 2013).

La tercera fase es el sistema de medición para la TC del hidrocarburo que consta de una válvula de compuerta con control motorizado MOV 1311C que permite el paso de hidrocarburo proveniente del sistema de filtrado. También se tiene una válvula de alivio de presión con regulador PSV 1311C para la seguridad del sistema. Para la medición se tiene incorporado un probador convencional FX 1311C que sirve para recalibrar in situ el medidor de caudal tipo turbina FE, el medidor tiene integrado un transmisor de flujo FT 1311D. Más adelante de la tubería se encuentra un transmisor de presión PT 1311D y una termocupla TW 1311E con transmisor de temperatura TT 1311E, que envían las señales recibidas hacia el computador de campo, para la evaluación de variables físicas del caudal, también se encuentra instalado un manómetro de presión PG 1311E. A la salida del sistema se acopló un indicador de temperatura TG 1311F y más adelante una válvula de control con actuador.

\section{Instrumentos para la automatización y control de la unidad LACT para medición dinámica de nafta y diésel}

En la Tabla 2 se muestran los instrumentos que integran el sistema de automatización y control de la unidad LACT para la medición dinámica de nafta y diésel en la estación Pozos Colorados.

En la parte superior izquierda de la Tabla 2 están los instrumentos de campo y panel, los cuales, interactúan con los instrumentos de campo de variable del proceso. En la parte superior derecha se establece la conectividad entre los instrumentos de campo con los instrumentos de control mediante el sistema de Supervisión, Control y Adquisición de Datos (SCADA). La Tabla 2 indica si el instrumento está montado en campo, mientras que su lazo de control lo identifica en el sistema de automatización.

Tabla 2. Instrumentos del sistema de automatización y control de la unidad LACT para medición dinámica de nafta y diésel en la estación Pozos Colorados

\begin{tabular}{|c|c|c|c|c|c|c|c|}
\hline \multirow[b]{2}{*}{$\begin{array}{c}\text { Instrumentos de campo y de } \\
\text { panel (I) }\end{array}$} & \multirow[b]{2}{*}{ Fase } & \multirow{2}{*}{$\begin{array}{l}\text { Instrumentos de campo de } \\
\text { variable del proceso } \\
\text { conectados a los } \\
\text { instrumentos de campo o } \\
\text { de panel }\end{array}$} & \multicolumn{3}{|c|}{$\begin{array}{c}\text { Supervisión, Control y } \\
\text { Adquisición de Datos (SCÁDA) }\end{array}$} & \multirow[b]{2}{*}{$\begin{array}{c}\text { Ubicado } \\
\text { en } \\
\text { Campo }\end{array}$} & \multirow[b]{2}{*}{$\begin{array}{l}\text { Lazo de } \\
\text { control }\end{array}$} \\
\hline & & & $\begin{array}{c}\text { Sistema } \\
\text { de } \\
\text { control }\end{array}$ & $\begin{array}{l}\text { Computador } \\
\text { de campo para } \\
\text { monitorear } \\
\text { variables de } \\
\text { medición }\end{array}$ & $\begin{array}{l}\text { Display con } \\
\text { conexión al } \\
\text { computador } \\
\text { de campo }\end{array}$ & & \\
\hline Válvula de bola 1 & FB & ZSV Sensor de posición & ZSV & & & I-ZSV & $1450 \mathrm{~A}$ \\
\hline Display indicador open & FB & $\begin{array}{l}\text { ZLO Indicador de posición } \\
\text { abierta de la válvula de bola } \\
1\end{array}$ & I-ZLO & & & & $1450 \mathrm{~A}$ \\
\hline
\end{tabular}




\begin{tabular}{|c|c|c|c|c|c|c|c|}
\hline Display indicador close & $\mathrm{FB}$ & $\begin{array}{l}\text { ZLC Indicador de posición } \\
\text { cerrada de la válvula de bola } \\
1\end{array}$ & I-ZLC & & & & $1450 \mathrm{~A}$ \\
\hline $\begin{array}{l}\text { Válvula de compuerta para } \\
\text { acople de instrumentos de baja } \\
\text { presión }\end{array}$ & FB & $\begin{array}{l}\text { PG Manómetro de baja } \\
\text { presión } \\
\text { PIT Transmisor indicador de } \\
\text { baja presión }\end{array}$ & PIT & & & $\begin{array}{l}\text { I-PG- } \\
\text { PIT }\end{array}$ & $\begin{array}{l}1450 \mathrm{~A} \\
1453 \mathrm{~A}\end{array}$ \\
\hline $\begin{array}{l}\text { Display indicador de baja } \\
\text { presión }\end{array}$ & FB & $\begin{array}{l}\text { PI Indicador de baja presión } \\
\text { I04 Alarma de baja presión }\end{array}$ & I-PI-I04 & & & & $\begin{array}{c}1453 \mathrm{~A} \\
04\end{array}$ \\
\hline Bomba de transferencia booster & FB & & & & & I & \\
\hline $\begin{array}{l}\text { Válvula de compuerta para } \\
\text { acople de instrumentos de alta } \\
\text { presión }\end{array}$ & $\mathrm{FB}$ & $\begin{array}{l}\text { PG Manómetro de alta } \\
\text { presión } \\
\text { PIT Transmisor indicador de } \\
\text { alta presión }\end{array}$ & PIT & & & $\begin{array}{l}\text { I-PG- } \\
\text { PIT }\end{array}$ & $\begin{array}{l}1452 \mathrm{~A} \\
1451 \mathrm{~A}\end{array}$ \\
\hline Display indicador de alta presión & $\mathrm{FB}$ & $\begin{array}{l}\text { PI Indicador de presión de } \\
\text { descarga }\end{array}$ & I-PI & & & & $1451 \mathrm{~A}$ \\
\hline $\begin{array}{l}\text { Sensor de dispersión térmica } \\
\text { para flujo FS }\end{array}$ & FB & & & & & I & $1450 \mathrm{~A}$ \\
\hline Display indicador de flujo & $\mathrm{FB}$ & $\begin{array}{l}\text { FAL Indicador de bajo } \\
\text { caudal } \\
\text { I04 Alarma de bajo caudal }\end{array}$ & $\begin{array}{l}\text { I-FAL- } \\
\text { I04 }\end{array}$ & & & & $\begin{array}{c}1450 \mathrm{~A} \\
04\end{array}$ \\
\hline $\begin{array}{l}\text { Válvula de compuerta para paso } \\
\text { de alivio de presión }\end{array}$ & FB & & & & & I & \\
\hline Válvula de alivio de alta presión & FB & $\begin{array}{l}\text { PSV Regulador de válvula } \\
\text { de alivio etapa de descarga }\end{array}$ & & & & I-PSV & 1440D \\
\hline Válvula de bola 2 salida de flujo & FB & ZSV Sensor de posición & ZSV & & & I-ZSV & $1451 \mathrm{~A}$ \\
\hline Display indicador open & FB & $\begin{array}{l}\text { ZLO Indicador de posición } \\
\text { abierta de la válvula de bola } \\
2\end{array}$ & I-ZLO & & & & $1451 \mathrm{~A}$ \\
\hline Display indicador open & FB & $\begin{array}{l}\text { ZLC Indicador de posición } \\
\text { cerrada de la válvula de } \\
\text { bola } 2\end{array}$ & I-ZLC & & & & $1451 \mathrm{~A}$ \\
\hline Filtro tipo canasta & $\mathrm{FF}$ & $\begin{array}{l}\text { PDIT Transmisor indicador } \\
\text { de presión diferencial }\end{array}$ & PDIT & & & I-PDIT & 1304 \\
\hline Válvula de compuerta & $\mathrm{FF}$ & $\begin{array}{l}\text { PI Indicador de presión de } \\
\text { filtrado }\end{array}$ & & & & I-PI & $1611 \mathrm{D}$ \\
\hline $\begin{array}{l}\text { Válvula de compuerta } \\
\text { motorizada }\end{array}$ & $\mathrm{FM}$ & $\begin{array}{l}\text { MOV Control de válvula } \\
\text { motorizada }\end{array}$ & MOV & & & I-MOV & $1311 \mathrm{C}$ \\
\hline $\begin{array}{l}\text { Válvula de compuerta para paso } \\
\text { de alivio de presión etapa } \\
\text { medición }\end{array}$ & $\mathrm{FM}$ & & & & & I & \\
\hline Válvula de alivio de presión & FM & $\begin{array}{l}\text { PSV Regulador de válvula } \\
\text { de alivio etapa medición }\end{array}$ & & & & I-PSV & $1311 \mathrm{C}$ \\
\hline $\begin{array}{l}\text { Probador convencional de } \\
\text { recalibración del medidor de } \\
\text { caudal }\end{array}$ & FM & $\begin{array}{l}\text { FX Medidor de flujo } \\
\text { comparativo }\end{array}$ & & & & I-FX & $1311 \mathrm{C}$ \\
\hline Medidor de caudal tipo turbina & FM & $\begin{array}{l}\text { FE Elemento primario de } \\
\text { medición de caudal } \\
\text { FT Transmisor de flujo }\end{array}$ & & FT & & I-FE-FT & 1311D \\
\hline $\begin{array}{l}\text { Válvula de compuerta para } \\
\text { acople de instrumento de } \\
\text { transmisión }\end{array}$ & FM & $\begin{array}{l}\text { PT Transmisor de presión de } \\
\text { caudal }\end{array}$ & & PT & & I-PT & 1311D \\
\hline $\begin{array}{l}\text { Válvula de compuerta para } \\
\text { acople de instrumento de } \\
\text { medición }\end{array}$ & FM & $\begin{array}{l}\text { PG Manómetro de presión de } \\
\text { caudal }\end{array}$ & & & & I-PG & $1311 \mathrm{E}$ \\
\hline $\begin{array}{l}\text { Termocupla de temperatura de } \\
\text { caudal TW }\end{array}$ & FM & $\begin{array}{l}\text { Transmisor de temperatura } \\
\text { TT }\end{array}$ & & I & & I-TT & $1311 \mathrm{E}$ \\
\hline Indicador de temperatura TG & FM & & & & & $\mathrm{I}$ & $1311 \mathrm{~F}$ \\
\hline $\begin{array}{l}\text { Computadoras de control de } \\
\text { variables físicas de caudal FC }\end{array}$ & FM & $\begin{array}{l}\text { TI Indicador de temperatura } \\
\text { PI Indicador de presión } \\
\text { FQR Registrador de flujo } \\
\text { FY Controlador de flujo } \\
\text { FIC Controlador indicador } \\
\text { de flujo }\end{array}$ & & & $\begin{array}{l}\text { TI-PI-FQR- } \\
\text { FY-FIC }\end{array}$ & I & \\
\hline $\begin{array}{l}\text { Display indicador de } \\
\text { temperatura }\end{array}$ & FM & $\begin{array}{l}\text { TI Indicador de temperatura } \\
\text { de flujo }\end{array}$ & I-TI & I-TI & & & 1311D \\
\hline Display indicador de presión & FM & $\begin{array}{l}\text { PI Indicador de presión de } \\
\text { flujo }\end{array}$ & I-PI & I-PI & & & 1311D \\
\hline $\begin{array}{l}\text { Display indicador de registro de } \\
\text { flujo }\end{array}$ & FM & $\begin{array}{l}\text { FQR Indicador de registro de } \\
\text { flujo }\end{array}$ & I-FQR & I-FQR & & & 1311D \\
\hline Display indicador de flujo & FM & FI Indicador de flujo & I-FI & I-FI & & & 1311D \\
\hline Válvula de control con actuador & FM & & & I & & I & \\
\hline
\end{tabular}

Fuente: Elaboración propia basada en la Figura 4 
Sistema de automatización en una unidad LACT para medición dinámica de petróleo en el complejo de producción y facilidades (CPF) Indillana de Petroamazonas EP

El campo Indianilla, ubicado en el cantón Shushufindi, pertenece al bloque 15 de la empresa pública (EP) ecuatoriana de exploración y explotación de hidrocarburos Petroamazonas. En este campo son extraídos una mezcla de petróleo, gas y agua, y enviados al complejo de producción y facilidades CPF Indianilla para su separación a partir de una serie de procesos. Una vez obtenido el petróleo es enviado a tanques de almacenamiento para posteriormente realizar su medición dinámica de flujo mediante la unidad LACT y enviarlo por oleoducto a la estación de transferencia de Shushufindi (Falcón, 2017). A partir del proyecto se detalló para esta investigación el diagrama de tuberías e instrumentación y los instrumentos para la automatización y control de la unidad LACT.

\section{Diagrama de tuberías e instrumentación (P\&ID) de la unidad LACT para medición dinámica de petróleo}

En la Figura 5 se muestra el P\&ID de la unidad LACT para la medición dinámica de caudal de petróleo, el diseño de la unidad es un estándar que la integran la fase de bombeo, la fase de muestreo y la fase de medición. El P\&ID sirve como guía para realizar los primeros pasos en la automatización y control de los elementos que conforman la unidad LACT, además de, permitir la correcta medición de caudal del hidrocarburo en el CPF Indianilla (Falcón, 2017).

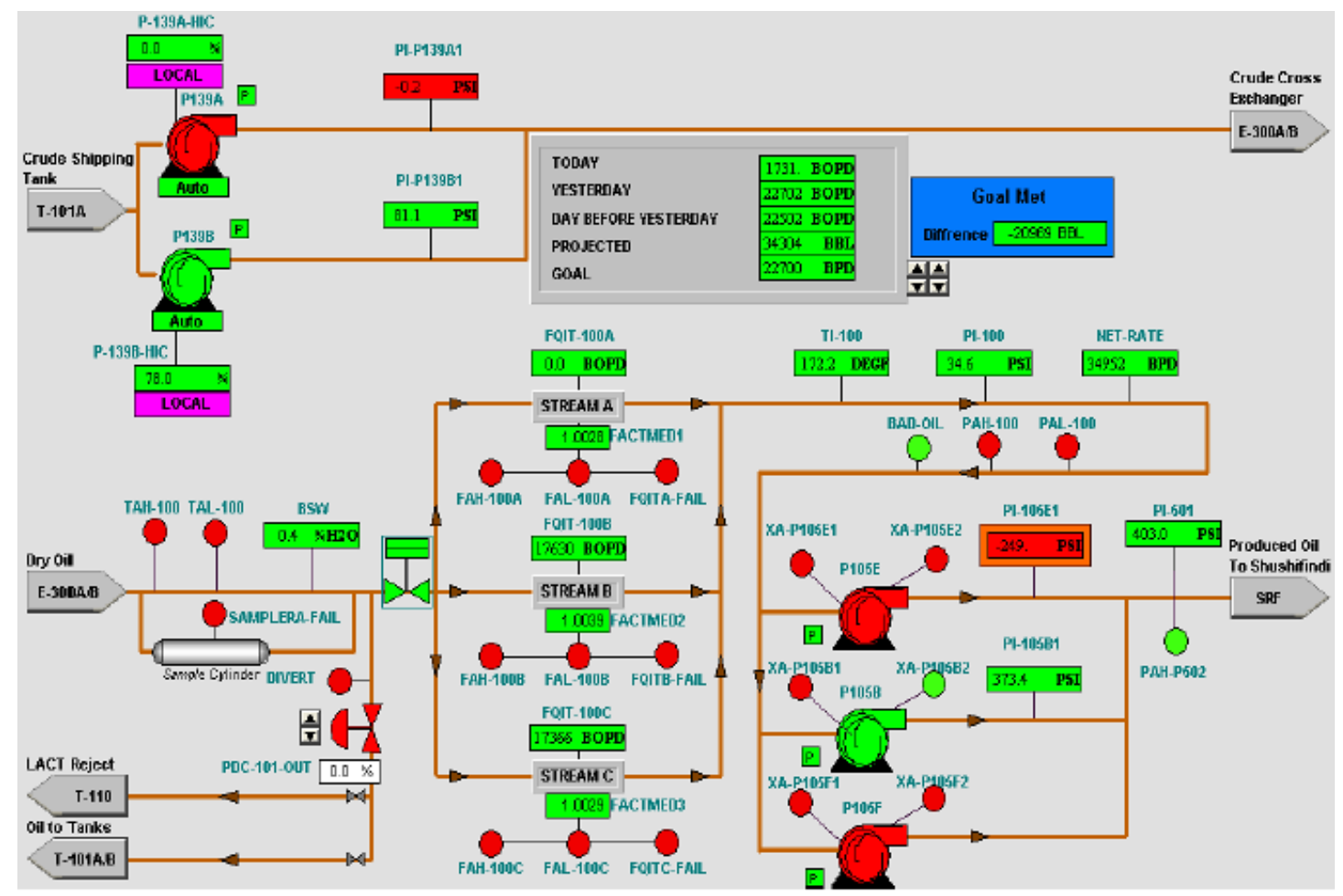

Figura 5. P\&ID de la unidad LACT para la medición dinámica de caudal de petróleo (Falcón, 2017) 
La primera fase es el sistema de bombeo, consta de bombas booster P139A y P139B que succionan el petróleo del tanque de almacenamiento y lo envía a los intercambiadores de calor A-300A/B, con el fin de reducir su temperatura para precautelar el medidor de flujo (Falcón, 2017).

La segunda fase es el sistema de muestreo, aquí se encuentra el dispositivo de muestreo automático (Sample Cylinder), en donde se recolecta un volumen específico de petróleo y lo analiza acorde a las necesidades del proceso de fiscalización (Falcón, 2017).

La tercera fase es el sistema de medición para la TC del hidrocarburo que consta de tres medidores de caudal que miden los barriles de petróleo por día (Barrels Oil Per Day, BOPD) que fluyen por la tubería (Falcón, 2017).

\section{Instrumentos para la automatización y control de la unidad LACT para medición dinámica de petróleo}

En la Tabla 3 se muestran los instrumentos que integran el sistema de automatización y control de la unidad LACT para la medición dinámica de petróleo en el complejo de producción y facilidades (CPF) Indillana de Petroamazonas EP.

En la parte superior izquierda de la Tabla 3 están los instrumentos de campo y panel, los cuales, interactúan con los instrumentos de campo de variable del proceso. En la parte superior derecha se establece la conectividad entre los instrumentos de campo con los instrumentos de control mediante el sistema de Supervisión, Control y Adquisición de Datos (SCADA). La Tabla 3 indica si el instrumento está montado en campo, mientras que su lazo de control lo identifica en el sistema de automatización.

Tabla 3. Instrumentos del sistema de automatización y control de la unidad LACT para medición dinámica de petróleo en el complejo de producción y facilidades (CPF) Indillana de Petroamazonas

\begin{tabular}{|c|c|c|c|c|c|}
\hline $\begin{array}{l}\text { Instrumentos de campo y de panel } \\
\text { (I) }\end{array}$ & Fase & $\begin{array}{l}\text { Instrumentos de variable del proceso } \\
\text { conectado con los instrumentos de } \\
\text { campo o de panel }\end{array}$ & $\begin{array}{l}\text { Sistema } \\
\text { de } \\
\text { control }\end{array}$ & $\begin{array}{l}\text { Ubicado en } \\
\text { Campo }\end{array}$ & $\begin{array}{l}\text { Lazo de } \\
\text { control }\end{array}$ \\
\hline $\begin{array}{l}\text { Bomba de transferencia booster } \\
\text { P139A }\end{array}$ & FB & & & I & \\
\hline Display indicador bomba P139A & FB & $\begin{array}{l}\text { HIC Sensor de transmisión de datos de la } \\
\text { bomba P139A }\end{array}$ & I-HIC & & P139A \\
\hline $\begin{array}{l}\text { Bomba de transferencia booster } \\
\text { P139B }\end{array}$ & FB & & & I & \\
\hline Display indicador bomba P139B & FB & $\begin{array}{l}\text { HIC Sensor de transmisión de datos de la } \\
\text { bomba P139B }\end{array}$ & I-HIC & & P139B \\
\hline $\begin{array}{l}\text { Display indicador de presión bomba } \\
\text { P139A }\end{array}$ & FB & $\begin{array}{l}\text { PT Transmisor de presión } \\
\text { PI indicador de presión }\end{array}$ & I-PI & I-PT & P139A1 \\
\hline $\begin{array}{l}\text { Display indicador de presión bomba } \\
\text { P139B }\end{array}$ & FB & $\begin{array}{l}\text { PT Transmisor de presión } \\
\text { PI indicador de presión }\end{array}$ & I-PI & I-PT & P139B1 \\
\hline Intercambiador de calor & FB & & & $\mathrm{I}$ & \\
\hline TAH Alarma de alta temperatura & FMU & & & I & 100 \\
\hline
\end{tabular}

Fuente: Elaboración propia basada en la Figura 5 
Tabla 3. Continuación

\begin{tabular}{|c|c|c|c|c|c|}
\hline $\begin{array}{l}\text { Instrumentos de campo y de panel } \\
\text { (I) }\end{array}$ & Fase & $\begin{array}{c}\text { Instrumentos de variable del proceso } \\
\text { conectado con los instrumentos de } \\
\text { campo o de panel }\end{array}$ & $\begin{array}{l}\text { Sistema de } \\
\text { control }\end{array}$ & $\begin{array}{l}\text { Ubicado en } \\
\text { Campo }\end{array}$ & $\begin{array}{l}\text { Lazo de } \\
\text { control }\end{array}$ \\
\hline TAL Alarma de baja temperatura & FMU & & & I & 100 \\
\hline Display indicador BSW & FMU & BSW Transmisor de agua libre y sedimentos & I-BSW & BSW & \\
\hline $\begin{array}{l}\text { Dispositivo de muestreo automático } \\
\text { (Sample Cylinder) }\end{array}$ & FMU & FAIL Alarma de muestra & & I-FAIL & FAIL \\
\hline Válvula de control con actuador & FM & & I & I & \\
\hline $\begin{array}{l}\text { Medidor de caudal (barriles de } \\
\text { petróleo por día) (STREAM A) }\end{array}$ & FM & $\begin{array}{l}\text { FT Transmisor de flujo } \\
\text { FACMED1 Factor de medición } \\
\text { FAH Alarma de alto flujo } \\
\text { FAL Alarma de bajo flujo } \\
\text { FQITA Alarma de indicación de cantidad de } \\
\text { flujo }\end{array}$ & $\begin{array}{l}\text { FT- } \\
\text { FACMED1 }\end{array}$ & $\begin{array}{l}\text { FT- } \\
\text { FACMED1- } \\
\text { FAH-FAL- } \\
\text { FQITA }\end{array}$ & $\begin{array}{l}100 \mathrm{~A} \\
100 \mathrm{~A} \\
100 \mathrm{~A} \\
100 \mathrm{~A} \\
100 \mathrm{~A}\end{array}$ \\
\hline $\begin{array}{l}\text { Display indicador de barriles de } \\
\text { petróleo por día (A) }\end{array}$ & FM & $\begin{array}{l}\text { FQI Indicador integrador de cantidad de } \\
\text { flujo }\end{array}$ & I-FQIT & & $100 \mathrm{~A}$ \\
\hline $\begin{array}{l}\text { Medidor de caudal (barriles de } \\
\text { petróleo por día) (STREAM B) }\end{array}$ & FM & $\begin{array}{l}\text { FT Transmisor de flujo } \\
\text { FACMED2 Factor de medición } \\
\text { FAH Alarma de alto flujo } \\
\text { FAL Alarma de bajo flujo } \\
\text { FQITB Alarma de indicación de cantidad de } \\
\text { flujo }\end{array}$ & $\begin{array}{l}\text { FT- } \\
\text { FACMED2 }\end{array}$ & $\begin{array}{l}\text { FT- } \\
\text { FACMED2- } \\
\text { FAH-FAL- } \\
\text { FQITA }\end{array}$ & $\begin{array}{l}100 \mathrm{~B} \\
100 \mathrm{~B} \\
100 \mathrm{~B} \\
100 \mathrm{~B} \\
100 \mathrm{~B}\end{array}$ \\
\hline $\begin{array}{l}\text { Display indicador de barriles de } \\
\text { petróleo por día (B) }\end{array}$ & FM & $\begin{array}{l}\text { FQI Indicador integrador de cantidad de } \\
\text { flujo }\end{array}$ & I-FQIT & & $100 \mathrm{~B}$ \\
\hline $\begin{array}{l}\text { Medidor de caudal (barriles de } \\
\text { petróleo por día) (STREAM C) }\end{array}$ & FM & $\begin{array}{l}\text { FT Transmisor de flujo } \\
\text { FACMED3 Factor de medición } \\
\text { FAH Alarma de alto flujo } \\
\text { FAL Alarma de bajo flujo } \\
\text { FQITC Alarma de indicación de cantidad de } \\
\text { flujo }\end{array}$ & $\begin{array}{l}\text { FT- } \\
\text { FACMED3 }\end{array}$ & $\begin{array}{l}\text { FT- } \\
\text { FACMED1- } \\
\text { FAH-FAL- } \\
\text { FQITA }\end{array}$ & $\begin{array}{l}100 \mathrm{C} \\
100 \mathrm{C} \\
100 \mathrm{C} \\
100 \mathrm{C} \\
100 \mathrm{C}\end{array}$ \\
\hline $\begin{array}{l}\text { Display indicador de barriles de } \\
\text { petróleo por día (B) }\end{array}$ & FM & $\begin{array}{l}\text { FQI Indicador integrador de cantidad de } \\
\text { flujo }\end{array}$ & I-FQIT & & $100 \mathrm{C}$ \\
\hline Transmisor de temperatura de flujo & FM & & I & I & \\
\hline $\begin{array}{l}\text { Display indicador de temperatura de } \\
\text { flujo }\end{array}$ & FM & TI Indicador de temperatura de flujo & I-TI & & \\
\hline Transmisor de presión de flujo & FM & & $\mathrm{I}$ & I & \\
\hline Display indicador de presión de flujo & FM & PI Indicador de presión de flujo & I-PI & & \\
\hline Transmisor de flujo & FM & & $\mathrm{I}$ & I & \\
\hline $\begin{array}{l}\text { Display indicador total de barriles de } \\
\text { petróleo por día a la salida del tubo }\end{array}$ & FM & FI Indicador de flujo & I-FI & & \\
\hline FB $=$ Fase de bombeo, FMU = Fase $\mathrm{d}$ & ue & o, FM= Fase de medición & & & \\
\hline
\end{tabular}

Fuente: Elaboración propia basada en la Figura 5

\section{Medidores de caudal para la industria de hidrocarburos}

El medidor de caudal es el instrumento de mayor importancia en una unidad LACT por tal motivo se hará referencia a varios de ellos que son utilizados los procesos de transferencia de custodia de hidrocarburos.

\section{Medidores de caudal ultrasónico Daniel 3417 para flujo en estaciones de gas}

El medidor de caudal ultrasónico Daniel modelo 3417, mostrado en la Figura 6, fue fabricado para maximizar el tiempo de actividad y ofrecer una precisión superior en transferencia de custodia de gas. Este medidor es lo más avanzado en verificación y validación de mediciones (Emerson, 2016). La medición del caudal se basa en el principio del tiempo de tránsito de los pulsos de ultrasonido que viajan diagonalmente a través de 
las paredes de la tubería, siendo la diferencia de los tiempos directamente proporcional al caudal.

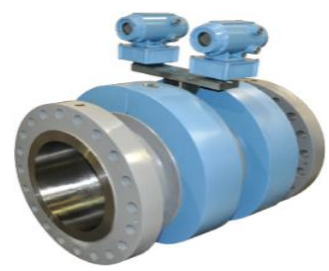

Figura 6. Medidor de caudal ultrasónico Daniel 3417 para flujo de gas (Emerson, 2016)

El software Daniel MeterLink permite a los operarios monitorear el medidor desde una PC o una computadora portátil, para un diagnóstico inmediato en las posibles alteraciones del caudal. El extenso registro del medidor permite observar tendencias en los dos medidores a lo largo del tiempo, ayudando a extender los ciclos de calibración (Emerson, 2016).

La Tabla 4 muestra los parámetros y especificaciones técnicas del medidor de caudal ultrasónico modelo Daniel 3417 diseñado y fabricado con respaldo de la compañía de tecnología Emerson Electric Co. Este medidor de caudal es extensamente utilizado en la industria petrolera, centrales térmicas, embarcaciones marinas, buques portacontenedores $\mathrm{y}$ en aplicaciones donde se tenga toda clase de procesos que involucre fluidos tipo gas.

Tabla 4. Parámetros del medidor de caudal ultrasónico Daniel 3417 (Emerson, 2016)

\begin{tabular}{r|l}
\hline Parámetros & Especificaciones técnicas \\
\hline Materiales & $\begin{array}{l}\text { Carcaza: Acero al carbono } \\
\text { Elementos internos: Acero inoxidable y aleación de aluminio }\end{array}$ \\
\hline Tamaños de la tubería & DN200 a DN1050 (8 in a 42 in) \\
\hline Tipos de fluido & Gas \\
\hline Linealidad & $\pm 0,1 \%$ de la lectura en todo el rango de calibración de caudal \\
\hline Repetibilidad & $\pm 0,05 \%$ de lectura para 5 a 100 fps $(1,5$ a $30,5 \mathrm{~m} / \mathrm{s})$ \\
\hline Rango de temperatura & Transductores T-21: $-20{ }^{\circ} \mathrm{C} \mathrm{a}+100{ }^{\circ} \mathrm{C}\left(-4{ }^{\circ} \mathrm{F} \mathrm{a}+21{ }^{\circ} \mathrm{F}\right)$ \\
operativa & Transductores T-22: $-50{ }^{\circ} \mathrm{C} \mathrm{a}+100{ }^{\circ} \mathrm{C}\left(-58{ }^{\circ} \mathrm{F} \mathrm{a}+212{ }^{\circ} \mathrm{F}\right)$ \\
\hline
\end{tabular}

Fuente: Elaboración propia basada en (Emerson, 2016)

\section{Medidor de caudal tipo turbina Daniel 1500 para flujo de diésel y nafta}

El medidor de caudal tipo turbina Daniel modelo 1500, mostrado en la Figura 7, es el mejor instrumento de medición de caudal volumétrico para transferencia de custodia de crudo o productos refinados como nafta y diésel. La medición del caudal se basa en el principio de la velocidad angular de las aspas de la turbina, esta velocidad es originada por la presión que ejerce el hidrocarburo sobre las aspas, siendo directamente proporcional al caudal que fluye por la tubería (Emerson, 2020). 


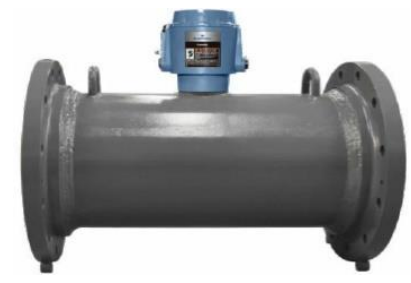

Figura 7. Medidor de caudal tipo turbina Daniel 1500 para flujo de hidrocarburos (Emerson, 2020)

La Tabla 5 muestra los parámetros y especificaciones técnicas del medidor de caudal tipo turbina Daniel 1500 diseñado y fabricado con respaldo de la compañía de tecnología Emerson Electric Co. Este medidor de caudal es extensamente utilizado en la industria de petróleo crudo, productos refinados de petróleo, carga y descarga de vagones y buques cisterna de hidrocarburos, almacenamiento flotante y descarga de hidrocarburos, entre otras.

Tabla 5. Parámetros del medidor de caudal tipo turbina Daniel 1500 (Emerson, 2020)

\begin{tabular}{|c|c|}
\hline Parámetros & Especificaciones técnicas \\
\hline Materiales & $\begin{array}{l}\text { Carcaza: Acero al carbono } \\
\text { Elementos internos: Acero inoxidable, aleación de } \\
\text { níquel y aleación de aluminio }\end{array}$ \\
\hline Tamaños de la tubería & DN25 a DN600 (1 in a 24 in $)$ \\
\hline Tipos de fluido & Petróleo crudo y derivados de petróleo \\
\hline Linealidad estándar & $\pm 0,25 \%(1$ in a 2,5 in $) ; \pm 0,15 \%$ ( 3 in a 24 in $)$ \\
\hline Repetibilidad & $\pm 0,02 \%$ \\
\hline $\begin{array}{r}\text { Rango de temperatura } \\
\text { operativa estándar }\end{array}$ & $\begin{array}{l}\text { Acero al carbono: }-29^{\circ} \mathrm{C} \mathrm{a}+60^{\circ} \mathrm{C}\left(-20^{\circ} \mathrm{F} \mathrm{a}+140^{\circ} \mathrm{F}\right) \\
\text { Acero inoxidable: }-40^{\circ} \mathrm{C} \mathrm{a}+60^{\circ} \mathrm{C}\left(-40^{\circ} \mathrm{F} \mathrm{a}+140^{\circ} \mathrm{F}\right)\end{array}$ \\
\hline
\end{tabular}

Fuente: Elaboración propia basada en (Emerson, 2020)

\section{Medidor de caudal másico tipo Coriolis SITRANS FCS300 para flujo de gas, diésel y nafta}

El medidor de caudal másico tipo Coriolis SITRANS FCS300 mostrado en la Figura 8, es actualmente utilizado para la medición de caudal en procesos de transferencia de custodia de gas, crudo o productos refinados como nafta y diésel. La medición del caudal se basa en la ley de movimiento de Coriolis (Siemens, 2021).

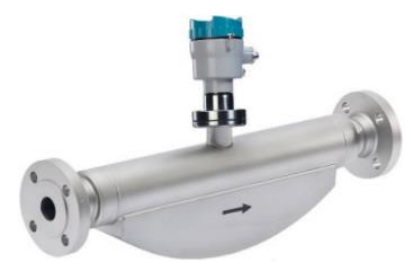

Figura 8. Medidor de caudal másico tipo Coriolis SITRANS FC300 para flujo de gas, diésel y nafta (Siemens, 2021) 
La Tabla 6 muestra los parámetros y especificaciones técnicas del medidor de caudal másico tipo Coriolis SITRANS FC300 diseñado y fabricado con tecnología de la compañía Siemens.

Tabla 6. Parámetros del medidor de caudal tipo Coriolis SITRANS FC300 (Siemens, 2021)

\begin{tabular}{r|l}
\hline Parámetros & Especificaciones técnicas \\
\hline Materiales & $\begin{array}{l}\text { Carcaza: Acero inoxidable } \\
\text { Elementos internos: Acero inoxidable, aleación } \\
\text { de níquel y aleación de aluminio }\end{array}$ \\
\hline Tamaños de la tubería & DN15 a DN150 (1/2 in a 6 in) \\
\hline Tipo de fluido & Petróleo crudo, derivados de petróleo, gas \\
\hline Linealidad estándar & $\begin{array}{l} \pm 0,2 \% \text { para líquidos } \\
\pm 0,4 \% \text { para gases }\end{array}$ \\
\hline $\begin{array}{r}\text { Rango de temperatura } \\
\text { operativa estándar }\end{array}$ & $-40{ }^{\circ} \mathrm{C} \mathrm{a}+70{ }^{\circ} \mathrm{C}\left(-40{ }^{\circ} \mathrm{F} \mathrm{a}+158^{\circ} \mathrm{F}\right)$ \\
\hline
\end{tabular}

Fuente: Elaboración propia basada en (Siemens, 2021)

\section{Red de comunicación industrial para los sistemas de automatización de unidades LACT}

La red de comunicación, para procesos de TC de hidrocarburos, permite al sistema de Supervisión, Control y Adquisición de Datos (SCADA) establecer la conectividad entre el Nivel 0, integrado por sensores, transmisores actuadores y todo dispositivo de campo, con el Nivel 1, integrado por controladores lógicos programables (PLC), sistemas de control numérico, tarjetas de microprocesadores o microcontroladores y computadores industriales. Las redes de comunicación industrial para TC de hidrocarburos, mediante unidades LACT, utilizan buses de campo para la transmisión de datos en forma digital bidireccional entre controladores y dispositivos de campo, lo que simplifica la instalación y operación de la instrumentación y equipos utilizados en los procesos de medición dinámica de hidrocarburos.

Actualmente, en los sistemas de automatización de la industria petrolera, incluyendo sistemas de automatización en unidades LACT, el bus de campo está reemplazando a las conexiones punto a punto que son sistemas de control aislados y centralizados y que funcionan con un bucle de corriente de 4 a $20 \mathrm{~mA}$. La tecnología de bus de campo es la red de comunicación industrial más sofisticada, ya que facilita el control distribuido entre dispositivos de campo y controladores. Para la establecer la conectividad entre la instrumentación de campo con los sistemas de control en unidades LACT, se la realiza mediante buses de campo como HART, ControlNet, DeviceNet, CAN Bus, Profibus y Foundation Field Bus, entre otros.

La Figura 9 muestra un sistema de automatización que integra la instrumentación de campo con los controladores mediante buses de campo. Este sistema de automatización puede ser utilizado en unidades LACT para la medición dinámica de hidrocarburos como los presentados en la Figura 3, Figura 4 y Figura 5. La Figura 9 muestra la conectividad de los elementos de campo como transmisores de presión, válvulas de control, medidores de flujo, transmisores de nivel y transmisores de temperatura de alta tecnología, con los 
controladores lógicos programables (PLC) y las unidades de periferia descentralizada, mediante redes de comunicación con buses de campo tipo PROFINET, PROFIBUS DP, HART protocol, PROFIBUS PA. El sistema SCADA integrado por la estación de servicio central, estación de ingeniería SIMATIC PCS 7 y la estación de mantenimiento SIMATIC PCS 7, realiza la gestión y control de cualquier sistema local o remoto, gracias a una interfaz gráfica que comunica al usuario con el sistema.

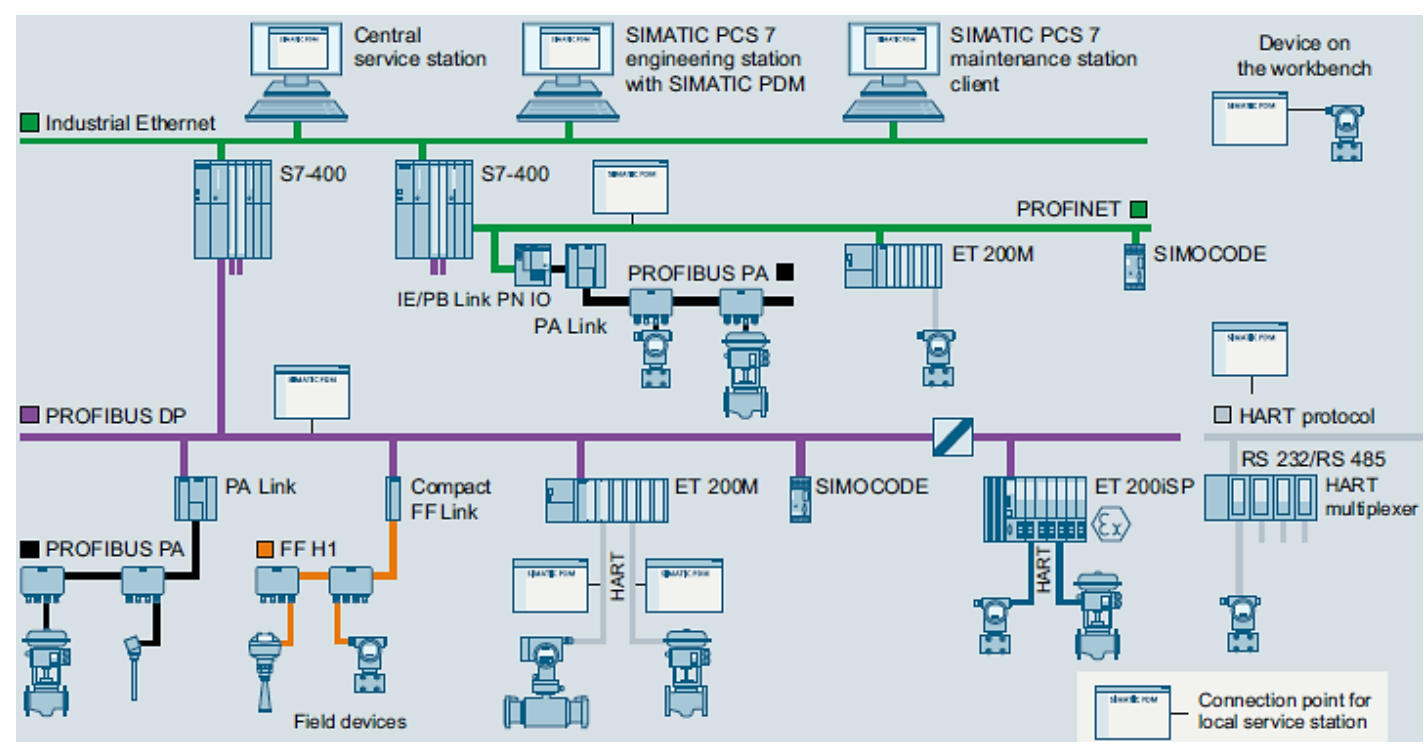

Figura 9. Sistema de automatización para la integración de la instrumentación de campo con los controladores mediante buses de campo (Siemens, 2021)

La Figura 10 muestra un sistema de automatización con aplicación basada en la nube SITRANS SAM IQ desarrollada por la compañía de tecnología Siemens. La aplicación Sitrans SAM (Smart Asset Management o Gestión de Activos Inteligentes) es una tecnología que en los últimos años está siendo implementada en el sector industrial, incluso en donde se requiera medición de flujo. En la Figura 10 se observa como el medidor de flujo y otros dispositivos de campo están interactuando con los dispositivos de control mediante buses de campo, mientras que la gestión del sistema de automatización lo realiza el sistema SCADA. Como parte del desarrollo de las redes informáticas la aplicación Sitrans SAM conecta la nube con el sistema de automatización.

El concepto de gestión de activos inteligentes, a través de la aplicación Sitrans SAM, permite el diagnóstico y monitorización de los datos en los dispositivos de campo, a través del navegador web Google Chrome instalado sea en ordenador de escritorio, tablet y smartphone. Esta aplicación es de gran ayuda en procesos productivos de manufactura o en procesos de producción de hidrocarburos en donde realiza medición dinámica con unidades LACT, ya que, mediante el big data se rastrea los instrumentos industriales que conforman la unidad LACT durante su ciclo de vida con el objetivo de su reparación o remplazo. Entre los principales beneficios que presenta esta aplicación en sistemas de automatización en unidades LACT es la de evitar futuras fallas de los dispositivos, reducción de costos de mantenimiento, mediciones confiables en dispositivos de 
medición, comunicación vía internet para diagnóstico y evaluación de instrumentos de campo, entre otros.

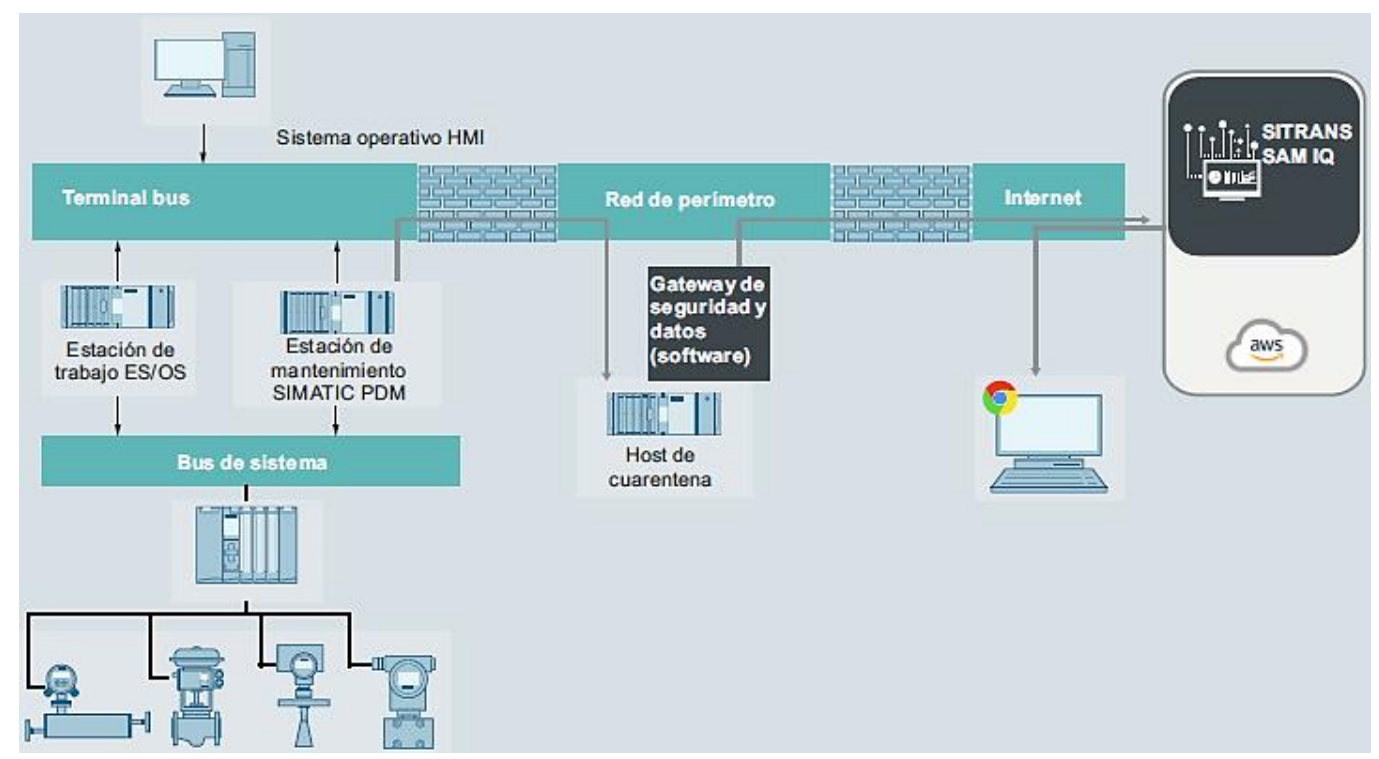

Figura 10. Sistema de automatización con aplicación basada en la nube SITRANS SAM IQ

(Siemens, 2021)

\section{Resultados obtenidos}

A partir del P\&ID se elaboró tablas sistematizadas que muestran los instrumentos industriales y su conectividad, mediante SCADA, con los instrumentos de control, como una guía para diseñar sistemas de automatización de unidades LACT para la medición dinámica de hidrocarburos en la industria petrolera.

Como resultado se ha presentado tres sistemas de automatización en unidades LACT para la medición dinámica de hidrocarburos en la industria petrolera, los mismos que constan de un diagrama de tuberías e instrumentación, instrumentos para la automatización y control, medidores de flujo y la red de comunicación mediante buses de campo.

Actualmente, los sistemas de automatización industrial, incluido los procesos de producción para TC de hidrocarburos, utilizan la tecnología de buses de campo para establecer la conectividad entre la instrumentación de campo y los controladores, entre los buses de campo se tiene HART, ControlNet, DeviceNet, CAN Bus, Profibus y Foundation Field Bus, tecnología que se basa en conceptos de automatización 4.0 y que se está implementando con más frecuencia en las industrias.

Los sistemas de automatización en unidades LACT para la medición dinámica de hidrocarburos, por lo general, tienen un diseño basado en tres fases. La primera es la fase de bombeo, la segunda es la fase de filtrado, muestreo y calidad, y la tercera es la fase de medición. 
El elemento principal de una unidad LACT es el medidor de flujo, su selección dependerá de factores como el tipo de hidrocarburo, la capacidad del instrumento para realizar medidas con exactitud, repetibilidad y linealidad estándar, así como, la compatibilidad de la marca con los instrumentos industriales.

\section{Conclusiones}

- La correcta medición dinámica de gas, nafta, diésel y petróleo, para el proceso de TC, por parte de los sistemas de automatización presentados, va a depender principalmente de la repetibilidad y linealidad estándar del medidor de flujo, el desarrollo de las redes informáticas y actualmente de las redes de comunicación mediante buses de campo. Estas dos últimos conceptos están dentro de lo que se denomina automatización 4.0.

- Un sistema de automatización de unidades LACT para la medición dinámica de hidrocarburos en la industria petrolera puede obtenerse de dos maneras. La primera, mediante un diseño y fabricación en el sitio donde se debe realizar la medición, como los expuestos en esta investigación, y la segunda, adquiriendo en el mercado una unidad ya fabricada conocida como patín de medición que son certificados para procesos de TC.

- El medidor de flujo es el principal instrumento en el proceso de transferencia de custodia de hidrocarburos, su capacidad de realizar medidas de linealidad estándar de alrededor de $\pm 0,2 \%$ y de repetibilidad de alrededor de $\pm 0,05 \%$, se debe al desarrollo tecnológico de empresas como Emerson, Siemens y otras más, que fabrican estos instrumentos.

- El propósito de fabricar y automatizar las unidades LACT es la medición de flujo de hidrocarburos con precisión y repetitividad de acuerdo a los estándares de la API.

\section{Referencias bibliográficas}

ABUIN GARCÍA OIL. (2016). LACT Unidades de medición fiscal. (Boletín CTS00). Venezuela.

Arequipa, H., \& Loyo, E. (2013). Estandarización de las inspecciones técnicas para sistemas de medición dinámica de hidrocarburos en las estaciones de producción del distrito amazónico (Tesis de Grado). UCE. Quito.

Emerson. (2016). Daniel ${ }^{\mathrm{TM}}$ modelo 3417 Medidor de caudal de gas ultrasónico redundante de cuatro rutas. USA: DAN-GUSM. Recuperado de https://www.emerson.com/documents/automation/hoja-de-datos-del-productodaniel-3417-4-4-dual-configuration-gas-ultrasonic-meter-es-es-176866.pdf 
Emerson. (2020). Caudalímetro de turbina de líquidos serie 1500 Daniel $^{\mathrm{TM}}$. USA. Recuperado de https://www.emerson.com/documents/automation/hoja-dedatos-del-producto-1500-series-daniel-liquid-turbine-meteres-7485828.pdf

Escobar-Díaz, A., Marín-Oviedo, L. E., \& Vacca-González, H. (2018). Instrumentación para sistemas automatizados de medición dinámica de hidrocarburos. Revista Ingeniería Solidaria, 14(26).

Falcón, D. (2017). Optimización del proceso de deshidratación del crudo en la planta de tratamiento del complejo de producción y facilidades Indillana (CPF) de Petroamazonas EP, bloque 15 en el año 2015. Rediseño del proceso de deshidratación del crudo en el año 2015 (Tesis de Maestría). UTC. Latacunga.

FMC Technologies. (2012). LACT Unit. (Bulletin TP0A016). USA: Smith Meter.

Jiménez, F., Rodriguez, O., \& Cárdenas, P. (2013). Ingeniería de detalle para ampliación y automatización del proceso de bombeo en pozos petroleros. Revista colombiana de tecnologías de avanzada (RCTA), 1(21), 27-36.

Lipták, B. G., \& Venczel, K. (Eds.). (2016). Instrument and Automation Engineers' Handbook Volume I Measurement and Safety. Florida: CRC Press.

Sánchez, R. (2010). Diseño de la automatización de los sistemas de medición estática y dinámica de combustible en el proceso de generación de una central termoeléctrica (Tesis de Grado). ESPE. Sangolquí.

Siemens. (2021). Productos para la instrumentación de procesos (Catálogo FI 01-2021). Alemania: Siemens AG. Recuperado de https://support.industry.siemens.com/cs/document/109745616/cat\%C3\%A1log o-fi-01-\%C2\%B7-2021-productos-para-la-instrumentaci\%C3\%B3n-deprocesos? $\mathrm{dti}=0 \& \mathrm{lc}=\mathrm{es}-\mathrm{WW}$

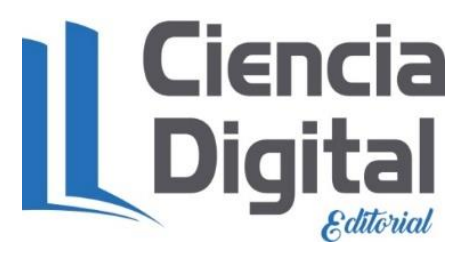




\section{PARA CITAR EL ARTÍCULO INDEXADO.}

Padilla Romero, A. D., Chamba Melo, S. C., Aguilera Flores, G. C., \& Anguaya Isama, H. F. . (2021). Sistemas de automatización en unidades LACT para la medición dinámica de hidrocarburos en la industria petrolera. AlfaPublicaciones, 3(3.1), 6-28. https://doi.org/10.33262/ap.v3i3.1.73

\section{LCiencia}

El artículo que se publica es de exclusiva responsabilidad de los autores y no necesariamente reflejan el pensamiento de la Revista Alfa Publicaciones.

El artículo queda en propiedad de la revista y, por tanto, su publicación parcial y/o total en otro medio tiene que ser autorizado por el director de la Revista Alfa Publicaciones.
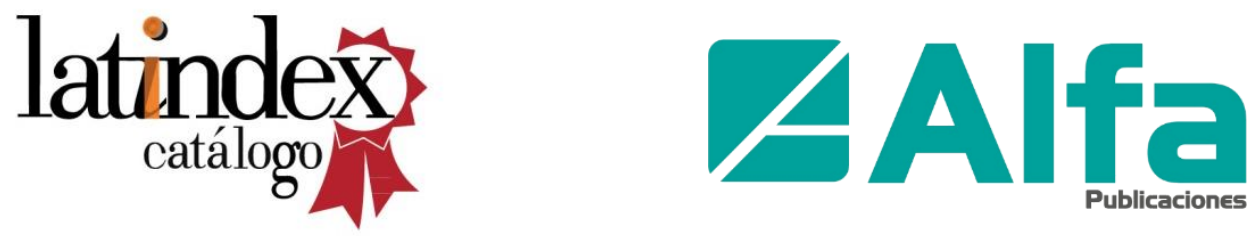